\title{
Computational efficiency of staggered Wilson fermions: A first look
}

\section{David H. Adams*}

Division of Mathematical Sciences, Nanyang Technological University, Singapore 637371

E-mail: dhadamsentu.edu.sg

\section{Dániel Nógrádi}

Institute for Theoretical Physics, Eötvös University, H-1117 Budapest, Hungary

E-mail: nogradi@bodri.elte.hu

\section{Andrii Petrashyk ${ }^{\dagger}$}

Division of Mathematical Sciences, Nanyang Technological University, Singapore 637371

E-mail: ap3115ecolumbia.edu

\section{Christian Zielinski}

Division of Mathematical Sciences, Nanyang Technological University, Singapore 637371

E-mail: zielinski@pmail.ntu.edu.sg

Results on the computational efficiency of 2-flavor staggered Wilson fermions compared to usual Wilson fermions in a quenched lattice QCD simulation on $16^{3} \times 32$ lattice at $\beta=6$ are reported. We compare the cost of inverting the Dirac matrix on a source by the conjugate gradient (CG) method for both of these fermion formulations, at the same pion masses, and without preconditioning. We find that the number of CG iterations required for convergence, averaged over the ensemble, is less by a factor of almost 2 for staggered Wilson fermions, with only a mild dependence on the pion mass. We also compute the condition number of the fermion matrix and find that it is less by a factor of 4 for staggered Wilson fermions. The cost per CG iteration, dominated by the cost of matrix-vector multiplication for the Dirac matrix, is known from previous work to be less by a factor 2-3 for staggered Wilson compared to usual Wilson fermions. Thus we conclude that staggered Wilson fermions are 4-6 times cheaper for inverting the Dirac matrix on a source in the quenched backgrounds of our study.

31st International Symposium on Lattice Field Theory - LATTICE 2013

July 29 - August 3, 2013

Mainz, Germany

\footnotetext{
* Speaker.

${ }^{\dagger}$ Current address: Physics Dept., Columbia University, New York, USA
} 


\section{Introduction}

Staggered Wilson fermions are a novel lattice fermion formulation constructed by adding a "Wilson term" to the staggered fermion action. Depending on the choice of this term, the number of fermion species (flavors) is reduced from 4 to 2 or 1 [1,2]. A key idea in this construction is to choose the Wilson term in such a way that the exact flavored chiral symmetry of staggered fermions becomes the unflavored chiral symmetry of staggered Wilson fermions, broken explicitly by the staggered Wilson term [1]. The theoretical properties are then similar to usual Wilson fermions, but with a one component lattice fermion field just like staggered fermions.

This development originated in the spectral flow approach to the staggered fermion index in [3], which led to a staggered version of the overlap Dirac operator describing 2 fermion species. The staggered Wilson fermion arose as the kernel in that operator [1] (see also [4] for further details). Another version of staggered Wilson fermions, describing 1 fermion species, was later proposed in [2]. The staggered Wilson terms in both cases are combinations of the flavored mass terms of Golterman and Smit, which lift the degeneracy of the 4 staggered fermion flavors [5].

The main practical motivation for considering staggered Wilson fermions is that they are potentially a more computationally efficient version of Wilson fermions, and can also be used as kernel for constructing staggered versions of the overlap and domain wall fermions [1] which should be cheaper than the expensive originals. The breaking of chiral symmetry is less for staggered Wilson fermions compared to usual Wilson fermions, as discussed later, which indicates that the improved chiral properties of domain wall and overlap fermions should be cheaper to achieve in the staggered versions. Also, as new lattice fermion formulations, the staggered versions of Wilson, domain wall and overlap fermions can be used for testing universality in lattice QCD.

Here we report on a first test of the computational efficiency of staggered Wilson fermions. We compare the cost (computation time) of inverting the Dirac matrix on a source for 2-flavor staggered Wilson and usual Wilson fermions in quenched lattice QCD with fixed physical volume and lattice spacing. The ratio of these computation times is our efficiency measure. To be meaningful, the comparison should be done at fixed values of a physical quantity. We take this to be the pion mass. I.e. our cost comparison for inverting the Dirac matrix is done with the staggered Wilson and usual Wilson fermions having different bare quark masses such that the pion masses are the same. Thus our efficiency measure is a function of the pion mass.

In the following we first discuss the theoretical background for staggered Wilson fermions, and then present the results of our numerical study. We conclude with remarks on prospects for the computational efficiency of staggered overlap and domain wall fermions. In particular we discuss implications of our results for the computational efficiency of staggered overlap fermions. The results give hope that the efficiency speed-up in thermalized backgrounds may be significantly greater on lattices of our size or larger compared to the modest speed-up by a factor 2-3 found in the numerical study of De Forcrand et al. on a smaller lattice [6].

\section{Theoretical Background}

At first sight, introducing a staggered Wilson term appears to be problematic since it breaks some of the staggered fermion symmetries. The concern in this situation is that new counterterms 
can arise which satisfy the remaining symmetries; these would then need to be included in the bare action from the beginning and fine-tuned to reproduce continuum QCD.

For the 2-flavor staggered Wilson fermion the situation turns out to be fortuitous: it breaks the 'shift' symmetries of the staggered fermion (corresponding to certain flavor symmetries in the continuum), but only one new counterterm of mass-dimension $\leq 4$ arises, and its effect on the 2 physical fermion species is simply a wavefunction renormalization [1]. Therefore it does not need to be included in the bare action, and no fine-tuning is required (besides the usual fine-tuning of the usual mass term that one also does for usual Wilson fermions).

However, the situation is worse for the 1-flavor staggered Wilson fermion of [2]. Besides breaking the shift symmetries, it also breaks lattice rotation symmetry. A subgroup of the latter survives, but it is not enough to prevent a new gluonic counterterm of mass-dimension 4 from arising [7]. This term needs to be included in the bare action and fine-tuned, thus reducing the attractiveness of the 1-flavor staggered Wilson fermion for practical use.

Besides the issue of broken symmetries, there is also at first sight a chirality problem for staggered Wilson fermions: The unflavored staggered version of $\gamma_{5}$ violates the property $\gamma_{5}^{2}=1$ by $O(a)$ effects, and also one does not have the $\gamma_{5}$-hermiticity property $\gamma_{5} D_{W} \gamma_{5}=D_{W}^{\dagger}$ of the usual Wilson Dirac operator $D_{W}$. The solution is to use the flavored $\gamma_{5}$ that gives the exact flavored chiral symmetry of staggered fermions as the unflavored $\gamma_{5}$ of the staggered Wilson fermion [1]. This is possible because the flavored $\gamma_{5}$ acts in an unflavored way on the physical fermion species of the staggered Wilson fermion in both the 2- and 1-flavor cases. The staggered Wilson Dirac operators are then $\gamma_{5}$-hermitian and have the other Wilson-like properties required to construct staggered versions of domain wall and overlap fermions [1].

A significant drawback of the 2-flavor staggered Wilson formulation is that the $\mathrm{SU}(2)$ vector and chiral symmetries of 2 flavors of usual Wilson fermions are broken by lattice effects, just like the SU(4) symmetries of the staggered fermion. However, the unbroken symmetries, which include all the flavored rotation symmetries, are still enough to impose some of the same consequences as the $\mathrm{SU}(2)$ symmetries. E.g. they are enough to ensure a degenerate triplet of pions [8].

On the other hand, regarding flavor-singlet chiral symmetry, 2-flavor staggered Wilson fermions have no disadvantage compared to usual Wilson fermions. Thus, for flavor-singlet physics, and in particular the challenge of high-precision computation of the $\eta^{\prime}$ mass, staggered Wilson fermions and the associated staggered versions of domain wall and overlap fermions offer increased computational efficiency with no significant theoretical drawbacks as a lattice formulation for the light $u$ and $d$ quarks. This also appears to be the case for their use to calculate bulk quantities in QCD thermodynamics. So these are at least two important arenas where we envisage that staggered Wilson-based fermions will be advantageous compared to usual Wilson-based fermions. For other challenges where flavored chiral symmetry plays a more important role, such as in the computation of hadronic matrix elements in weak interaction processes, it remains to be seen whether or not staggered Wilson-based fermions are advantageous compared to currently used lattice fermions. This will depend both on how computationally efficient the staggered Wilson-based fermions are, and how severe the consequences of their SU(2) flavor symmetry breaking turns out to be.

We omit the explicit expression for the 2-flavor staggered Wilson Dirac operator here. It can be found in [1]. A detailed treatment of the theoretical aspects discussed here, and related aspects, is currently in preparation [7]. 


\section{Numerical Results}

Our quenched simulations were done on the $16^{3} \times 32$ lattice with 200 configurations generated at $\beta=6$, using the Chroma/QDP software for lattice QCD [9] (we hacked the asqtad staggered fermion code there to make the code for staggered Wilson fermions).

Our results for the pion mass as a function of the bare quark mass are shown in Fig. 1(a). A check on the validity of the staggered Wilson formulation is that it exhibits a linear relation $m_{\pi}^{2} \sim m$ in accordance with Chiral Perturbation Theory. Significantly, the additive mass renormalization is seen to be less for staggered Wilson compared to usual Wilson fermions. Similar results for the pion mass were earlier obtained independently by us and by De Forcrand et al. [6, 10]. Our "pion" operator in the staggered Wilson case is the same as the one described in [6].

Knowing the relations between pion mass and the bare quark mass, we can proceed to measure the costs of inverting the staggered Wilson and usual Wilson Dirac matrices on a source $\chi$ at the same pion mass. This was done by solving

$$
(D+m)^{\dagger}(D+m) \psi=(D+m)^{\dagger} \chi^{(\alpha)} .
$$

using the conjugate gradient (CG) method, without preconditioning ${ }^{1}$, with $\chi^{(\alpha)}$ being the point source at the origin with (spin and) color component $\alpha$. We averaged the cost over the different $\alpha$ 's (3 sources for staggered Wilson vs 12 for usual Wilson). Hence our results also give a cost comparison for computing the fermion propagator matrix for staggered Wilson and usual Wilson fermions.

The cost (computation time) ratio decomposes as

$$
\frac{\operatorname{cost}(W)}{\operatorname{cost}(S W)}=\left(\frac{i \operatorname{ters}(W)}{i \operatorname{iters}(S W)}\right) \times\left(\frac{\operatorname{cost} \operatorname{per} \operatorname{iter}(W)}{\operatorname{cost} \operatorname{per} i \operatorname{ter}(S W)}\right)
$$

where ' $W$ ' and ' $S W$ ' refers to Wilson and staggered Wilson, respectively, and 'iters' is the number of $\mathrm{CG}$ iterations for convergence with a given $\mathrm{CG}$ residual $\varepsilon$. When considering the fermion propagator, an extra factor 4 should be included on the right-hand side because of the 12 vs 3 sources for the usual Wilson vs staggered Wilson case. But this should not be included when using (3.2) as an estimate of the cost ratio for generating configurations in dynamical fermion simulations of lattice QCD, which is the main quantity of interest for ascertaining the computational efficiency of staggered Wilson fermions.

Our results for the CG iterations ratio, i.e. the first ratio on the right-hand side of (3.2), averaged over the configurations of our ensemble, are shown in Fig. 1(b) for a selection of our smaller pion masses. The number of CG iterations required with staggered Wilson fermions is seen to be less by almost a factor 2 compared to usual Wilson fermions. Furthermore, the iterations ratio has only a mild dependence on the pion mass, even though the number of CG iterations in the staggered Wilson and usual Wilson cases both depend strongly on it. The results for the two lowest pion masses are less reliable - we noticed some instability in the CG computations for these cases, affecting the number of iterations required for convergence.

\footnotetext{
${ }^{1}$ Preconditioning speeds up the computation for Wilson fermions by more than a factor 2 . It can also be done for staggered Wilson fermions, but we did not implement it yet, so the cost comparison here is without preconditioning.
} 

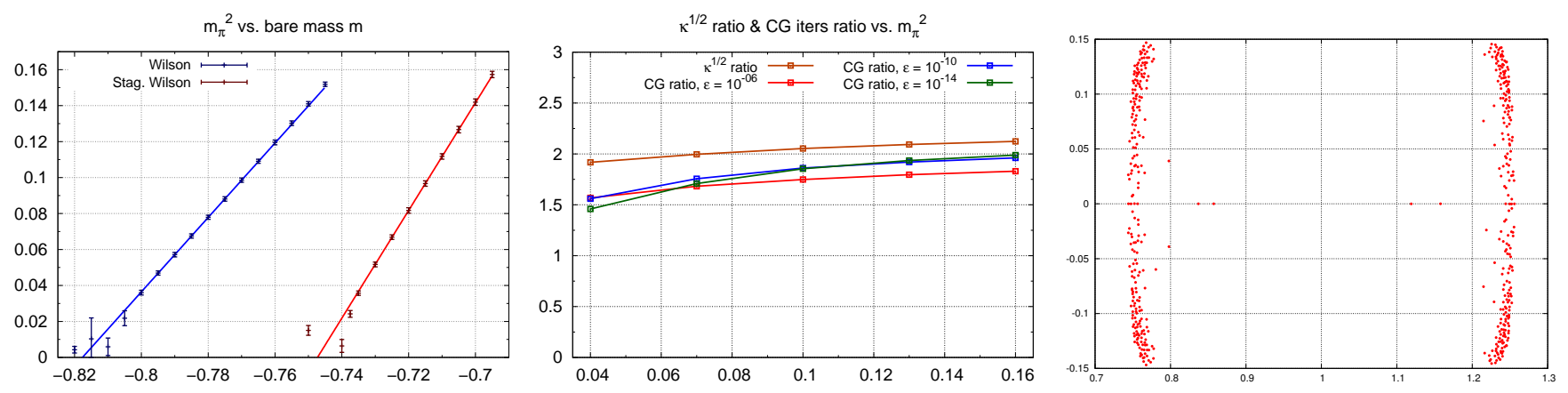

Figure 1: (a) Left: $m_{\pi}^{2}$ as a function of the bare quark mass $m$ in lattice units. Straight lines fitted through the data points with $0.05<m_{\pi}^{2}<0.1$. (b) Middle: averaged $\kappa^{1 / 2}$ ratio and CG iterations ratios as functions of $m_{\pi}^{2}$. Data joined by lines to guide the eye. (c) Right: The low-lying staggered Wilson eigenvalue spectrum in one of our backgrounds.

A related quantity of interest is the ratio $\kappa_{W}^{1 / 2} / \kappa_{S W}^{1 / 2}$, where $\kappa=\lambda_{\max } / \lambda_{\min }$ is the condition number of the fermion matrix $(D+m)^{\dagger}(D+m)$ in (3.1). The $\kappa^{1 / 2}$ ratio gives an estimate of the CG iterations ratio, which is expected to be better when the CG residual $\varepsilon$ is smaller. (See, e.g., [11].) We computed the condition number as a function of the pion mass in both the staggered Wilson and usual Wilson cases, and our results for the $\kappa^{1 / 2}$ ratio are also shown in Fig. 1(b). They are seen to agree approximately with the CG iterations ratios, with better agreement for smaller $\varepsilon$ as expected, except for the smaller pion masses where the above-mentioned CG instabilities occur.

The $\kappa^{1 / 2}$ ratio $\approx 2$ in Fig. $1(\mathrm{~b})$ can be compared with the free field case with same bare mass $m$ for both formulations: $\kappa_{W, \text { free }}^{1 / 2} / \kappa_{S W, \text { free }}^{1 / 2} \stackrel{m \rightarrow 0}{=} 8 / \sqrt{5} \approx 3.58$.

Regarding the second ratio in (3.2), the cost per CG iteration is dominated by the cost of matrix-vector multiplication with the fermion matrix $(D+m)^{\dagger}(D+m)$. It can be estimated as being proportional to the number of floating point operations required (this is only a rough first estimate though, since it does not take memory bandwidth into account). Consequently, for the ratio we have the estimate

$$
\frac{\text { cost per iter }(W)}{\text { cost per iter }(S W)} \approx 4 \times \frac{\text { flops }(W)}{\text { flops }(S W)}=4 \times \frac{1392}{1743} \approx 3.2 .
$$

Here 'flops' denotes the number of floating point operations per lattice site for matrix-vector multiplication with the lattice Dirac matrix $D$. We have plugged in the known value 1392 for the usual Wilson case, and the value 1743 that we find for the staggered Wilson $D$. The factor 4 after the equality in (3.3) is because the usual Wilson Dirac matrix is 4 times larger than the staggered Wilson one.

These numbers can be roughly understood as follows [6]: The staggered Wilson action couples each lattice site to $8+16$ other sites, whereas in the usual Wilson case it is $8 \times 2$ (the factor 2 is for the 2 Dirac spin components after the spin projections). Including the above-mentioned factor 4 , we then get the ratio estimate $4 \times(8 \times 2) /(8+16) \approx 2.67$ [6]. This is slightly smaller than in (3.3) because the small but non-negligible cost of spin decomposition and reconstruction in the spin projection trick has not taken into account.

A speed-up factor of order 2 for the matrix-vector multiplication in the staggered Wilson case was explicitly found in the numerical study of [6]. In light of this and the estimate (3.3), we assume 
the achievable speed-up factor to be 2-3. (We are far from achieving this speed-up in our own study, but that is no doubt due to shortcomings in our implementation of staggered Wilson fermions in Chroma. We hope to improve it in future.) Combining this with the speed-up factor $\approx 2$ for the number of CG iterations, we get an estimated speed-up factor of 4-6 for inverting the Dirac matrix on a source in the staggered Wilson case.

Finally, in Fig. 1(c) we show the low-lying spectrum of the staggered Wilson Dirac operator in one of the gauge field backgrounds of our ensemble. The situation is clearly better than in the small $8^{4}$ lattice results of [6]. E.g. the separation between the physical and doubler branches is $\sim 0.5$ in our case compared to $\sim 0.3$ in [6]. (See the 2 nd panel in Fig. 5 of the first article in [6].)

\section{Conclusions}

The estimated speed-up factor of 4-6 in the staggered Wilson case for inverting the Dirac matrix on a source gives tentative encouragement for the prospects of cheaper lattice QCD simulations with dynamical staggered Wilson fermions. However, it should be remembered that this is only a quenched exploratory study. It should be followed up in future by systematic studies of the computational efficiency in full QCD simulations, with $O(a)$ improvement of staggered Wilson fermions and using the HISQ version of the usual staggered part of the action to reduce $O\left(a^{2}\right)$ effects, and with smeared links, so that a realistic comparison with presently used improved Wilson fermions can be made. $O(a)$ improvement for staggered Wilson fermions via a version of the clover term has been done and will be reported elsewhere [7]. A different proposal for $O(a)$ improvement was recently studied, along with smearing, in [12].

On the other hand, our results give a complete solution to the problem of estimating the cost ratio for computing the fermion propagator in our quenched study. An extra factor 4 should be included in (3.2) for this, so we get an estimated speed-up factor of between 16-24 for computing the fermion propagator with staggered Wilson fermions.

The results here also have positive implications for the potential efficiency of staggered versions of domain wall and overlap fermions. They add to the evidence that staggered Wilson fermions are more chiral than usual Wilson fermions, as discussed below. The cost of overlap fermions is expected to be reduced when a more chiral kernel is used [13], and in the case of staggered fermions one can hope to achieve the same level of approximate chirality with a smaller 5th dimensional lattice size.

The more chiral nature of staggered Wilson fermions is strikingly clear in the free field case where the spectrum is close to the Ginsparg-Wilson (GW) circle [6]. In the interacting case, spectrum computations in thermalized backgrounds on a small $8^{4}$ lattice found that the spectrum collapses away from the GW circle into a vertical strip, with the physical branch becoming diffuse and approaching the doubler branch [6]. This was attributed to large fluctuations in the 4-link staggered Wilson term. However, our present results indicate that the chiral GW-like nature of staggered Wilson fermions does persist in the interacting case on larger lattices. If it did not, the condition number of the fermion matrix would be expected to be larger in the staggered Wilson case, due to the diffuse spectrum giving rise to near zero eigenvalues when the pion mass is small. But we found here that the condition number is smaller by a factor 4 for staggered Wilson fermions, cf. Fig. 1(b). The better-behaved spectrum in Fig. 1(c), and the smaller additive mass renormaliza- 
tion for staggered Wilson fermions in Fig. 1(a), is further evidence that the chiral GW-like nature persists in the thermalized backgrounds of our study.

The preceding has implications when assessing the potential of reduced cost for staggered overlap fermions. The computational cost of staggered overlap fermions vs usual overlap fermions for inverting the Dirac matrix on a source was investigated on a $12^{4}$ lattice in [6]. In the free field case, a large speed-up factor of almost 10 was found, confirming the expectations discussed above for reduced cost of overlap fermions when a more chiral kernel is used. However, in thermalized $\beta=6$ backgrounds the speed-up factor was found to be dramatically reduced to 2-3 [6]. This was attributed to the aforementioned ruining of the GW-like nature of the staggered Wilson kernel in the interacting case. But in the present case, on our larger lattice, our results indicate, as discussed above, that the GW-like nature survives to a reasonable extent. Thus one can hope for much better efficiency of staggered overlap fermions on our lattice and larger lattices in the interacting case. In this way our results suggest that the modest speed-up factor 2-3 found in [6] is not indicative of the true potential of reduced cost with staggered overlap fermions.

We hope to clarify the situation in the near future by repeating the present study for staggered overlap fermions (on our larger $16^{3} \times 32$ lattice), so as to determine the cost ratio as a function of the pion mass. Similar studies for staggered domain wall fermions are also planned.

Acknowledgments. D.A. and A.P. thank the Yukawa Institute, Kyoto, for hospitality and support at the workshop "New Types of Fermions on the Lattice", which spurred on this work. D.A. thanks Philippe de Forcrand for feedback on a previous version of the paper. D.A. is supported by AcRF grant RG61/10. D.N. is supported by the EU under grant (FP7/2007-2013)/ERC No 208740 and by OTKA under grant OTKA-NF-104034.

\section{References}

[1] D.H. Adams, Phys. Lett. B 699 (2011) 394 [arXiv:1008.2833]

[2] C. Hoelbling, Phys. Lett. B 696 (2011) 422 [arXiv:1009.5362]

[3] D.H. Adams, Phys. Rev. Lett. 104:141602 (2010) [arXiv:0912.2850]

[4] D.H. Adams, PoS LATTICE2010 (2010) 073 [arXiv:1103.6191]

[5] M.F.L. Golterman and J. Smit, Nucl. Phys. B 245 (1984) 61

[6] Ph. de Forcrand, A. Kurkela and M. Panero, PoS LATTICE2010 (2010) 080 [arXiv:1102.1000]; JHEP 1204 (2012) 142 [arXiv:1202.1867]

[7] D.H. Adams, unpublished work (articles in preparation).

[8] S. Sharpe, talk at Yukawa Institute Workshop "New Types of Fermions on the Lattice", Kyoto 2012.

[9] R.G. Edwards and B. Joo, Nucl. Phys. Proc. Suppl. 140 (2005) 832 [hep-lat/0409003]

[10] D. Adams, talk at Yukawa Institute Workshop "New Types of Fermions on the Lattice", Kyoto 2012.

[11] J.R. Shewchuk, "An Introduction to the Conjugate Gradient Method Without Agonizing Pain." http://www.cs.cmu.edu/ quake-papers/painless-conjugate-gradient.pdf

[12] S. Durr, Phys. Rev. D87 (2013) 114501 [arXiv:1302.0773]

[13] W. Bietenholz, Eur. Phys. J. C6 (1999) 537 [hep-lat/9803023] 